\title{
Mucormicose rinocerebral: apresentação de caso com sobrevida e revisão de literatura
}

\author{
Sandra Doria Xavier ${ }^{1}$, Gustavo Polacow Korn ${ }^{1}$, \\ Lídio Granato²
}

\section{Resumo / Summary}

\section{A} (1) Forma invasiva, que se divide em aguda e crônica; (2) Forma não-invasiva, que consta da bola fúngica (micetoma) e a sinusite fúngica alérgica. 0 desenvolvimento das diferentes formas de sinusite fúngica depende do estado imunológico do paciente, sendo que a forma invasiva aguda ocorre na grande maioria das vezes em imunodeprimidos. Os autores apresentam um caso de uma paciente do sexo feminino, diabética que iniciou quadro clínico com as características de sinusite fúngica invasiva, com febre alta, comprometimento ocular que evoluiu para amaurose à esquerda. Submetida à cirurgia, não apresentou boa evolução, com a manutenção de fístula nasocutânea. Após a introdução de medicação antifúngica (anfotericina B), seu quadro clínico estabilizou-se, com cessação da rinorréia fétida, febre e sinais flogísticos periorbitais. No exame anatomo-patológico foi encontrado mucosa revestida por epitélio respiratório com processo inflamatório tendo em meio hifas septadas com diagnóstico final de zigomicose. Após um ano de total desaparecimento dos sintomas, a paciente foi encaminhada ao Serviço de Cirurgia Plástica onde foi realizada cirurgia estética na região naso orbitária esquerda, estando a paciente atualmente satisfeita com aspecto facial.

\section{Rhinocerebral mucormycose: case presentation and literature review}

Palavras-chave: mucormicose, zigomicose, mucormicose rinocerebral, infecções fúngicas.

Key words: mucormycosis, zygomycosis, rinocerebral mucormycosis, sinusal fungal infections. ungal sinusitis are divided in two big groups: (1) Invasive form that is subdivided in acute and chronic; (2) Noninvasive form that is subdivided in mycetoma form (fungal ball) and allergic form. The development of one of these forms depends on the immunologic relationship of the fungus to the host, as invasive form occurs in most of the cases in immunocompromised hosts. It is reported a diabetic female, with suspected fungi sinusitis with clinical invasive features at its very beginning displaying high fever and left eye involvement that lead to amaurosis. A left orbital surgical drainage followed by sinusectomy resulted in a nasocutaneous fistula, that stabilized when antifungal treatment was instituted, with no more rinorrhea, fever and periorbital inflammatory signs. As improvement progressed the nasocutaneous fistula was closed under general anesthesia. On histopatology, respiratory epithelium with inflammatory process and septated hyphae was found, that disclosed zygomycosis. After one year of no patient's symptoms, she started to be followed by facial surgeons who did a naso-orbital esthetics surgery with a high patient's satisfaction.

\footnotetext{
${ }^{1}$ Residentes do 30 ano do Departamento de Otorrinolaringologia da Santa Casa de São Paulo. 


\section{INTRODUÇÃO}

As sinusites fúngicas são divididas simplificadamente em 2 grandes grupos ${ }^{1}$, a saber: (1) forma invasiva, que se divide em aguda e crônica; (2) forma não-invasiva, que se divide em bola fúngica (micetoma) e a sinusite fúngica alérgica. A análise histológica dos tecidos envolvidos, como a presença ou ausência de invasão vascular, é a base para a diferenciação entre as formas invasiva e não-invasiva. 0 desenvolvimento das diferentes formas de sinusite fúngica depende inteiramente do estado imunológico do paciente, sendo a forma invasiva aguda ou crônica ocorrendo na grande maioria das vezes em imunodeprimidos, a bola fúngica e a sinusite fúngica alérgica em atópicos. A classificação é, deste modo, baseada na resposta imunológica do hospedeiro frente ao fungo em questão ${ }^{2}$.

$\mathrm{Na}$ forma invasiva crônica ou também chamada "indolente", o tempo de evolução da doença é maior que quatro semanas o que a faz diferir da invasiva aguda, em que o tempo de evolução é menor que quatro semanas ${ }^{3}$. Além disso, como já previamente salientado, o hospedeiro é geralmente imunocompetente, sendo o Aspergillus o patógeno mais comum. $\mathrm{Na}$ tomografia computadorizada, pode-se observar velamento uni ou bilateral dos seios da face, com focos de hiperatenuação sugestivo de colonização fúngica ${ }^{4}$. Seu tratamento é feito com debridamento cirúrgico e os antifúngicos são necessários somente se houver invasão intracraniana ou de órbita ${ }^{1}$.

O micetoma ou bola fúngica caracteriza-se pelo comprometimento geralmente unilateral de um seio da face na maioria das vezes por Aspergillus, sendo o seio maxilar 0 mais freqüentemente acometido, seguido pelo seio esfenoidal. A sintomatologia mimetiza sinusite bacteriana, mas o paciente pode ser assintomátic $0^{5}$. Na tomografia computadorizada dos seios da face, observa-se velamento de unilateral de um seio da face com focos de hiperatenuação, que representam calcificações ou hifas ${ }^{5}$. Instala-se em hospedeiro imunocompetente, geralmente resolvida com debridamento cirúrgico e aeração sinusal, sendo a recorrência rara ${ }^{1}$.

A sinusite fúngica alérgica, por sua vez, é a forma mais comum de infecção fúngica sinusal ${ }^{6}$. É uma forma crônica que se desenvolve em indivíduos atópicos, com sintomas semelhantes aos de uma sinusite crônica bacteriana, muitas vezes com história de polipose nasal ${ }^{6}$. Bent e Kuhn ${ }^{7}$ desenvolveram o seguinte critério diagnóstico para sinusite fúngica alérgica: (1) hipersensibilidade tipo I, (2) pólipos nasais, (3) achados tomográficos característicos, com focos de hiperatenuação devido à mucina alérgica e com destruição óssea presente em 30-50\% dos $\operatorname{casos}^{1}$, (4) cultura para fungos positiva, (5) mucina alérgica com fungos e sem sinal de invasão tecidual. A mucina alérgica é caracterizada por eosinófilos, cristais de Charcot-Leyden e hifas ${ }^{6}$. Os fungos mais freqüentemente envolvidos neste tipo de sinusite fúngica são o Bipolaris, Curvularia e Alternaria ${ }^{3}$. 0 tratamento inclui debridamento cinúrgico e o uso de antifúngicos fica reservado para casos de invasão tecidual ${ }^{1}$.
A sinusite fúngica invasiva aguda acomete, na grande maioria das vezes, indivíduos imunodep rimidos, tendo como principais agentes etiológicos os fungos Mucor e Aspergillus, entre outros ${ }^{1}$. 0 termo mucormicose é usado para se referir a toda infecção fúngica causada por fungo da classe Zygomycetes e ordem Mucorales, sendo o representante mais comum o Rhizopus oryzae, que é responsável por $60 \%$ de todas as formas de mucormicose, além do Mucor, e Absidia ${ }^{3}$.

Qualquer tipo de imunodeficiência pode levar ao aparecimento da mucormicose, porém a cetoacidose diabética é o desencadeante mais freqüente ${ }^{3}$. Embora muito mais comum em pacientes com deficiência imunológica, a mucormicose pode afetar indivíduos sãos, como relataram Santana et al. ${ }^{8}$.

Mucormicose pode se apresentar em diferentes formas clínicas como pulmonar, gastrointestinal, no sistema nervoso central, cutânea e sistêmica, mas a forma mais comum de apresentação é a forma rinocerebral ${ }^{9}$.

Yohia et al. ${ }^{10}$ revisaram 208 casos de mucormicose rinocerebral entre 1970 e 1993 e encontraram como principais sintomas: febre (44\%), úlcera nasal ou necrose (38\%), edema periorbital ou facial (34\%), diminuição da acuidade visual (30\%), oftalmoplegia (29\%), sinusite (26\%) e cefaléia (25\%). Na verdade, com qualquer doente imunodep rimido, principalmente com cetoacidose diabética, com sintomas de rinossinusite sem melhora com tratamento antibiótico convencional, deve-se ter em mente o diagnóstico de mucormicose.

0 diagnóstico histológico da mucormicose baseia-se no achado de hifas não septadas ou com poucas septações, ao contrário, por exemplo, do Aspergillus, que sua hifa é mais estreita e freqüentemente septada com ângulo de $45^{05}$.

A tomografia computadorizada de seios da face pode mostrar invasão tecidual e necrose, erosão óssea e trombose de seio cavernoso. A ressonância nuclear magnética, com ou sem gadolíneo, é o melhor método para avaliar alterações precoces nos vasos como trombose de artéria carótida e do seio cavernoso. A ressonância também é o melhor meio de estudo de eventual extensão intracraniana ${ }^{11}$.

0 debridamento cirúrgico tem como principal objetivo remover o máximo de tecido desvitalizado possível além de estabelecer adequada drenagem sinusal ${ }^{12}$. Em anos recentes, cirurgia endoscópica como modo de tratamento cirúrgico da mucormicose vem ganhando popularidade ${ }^{13}$. No estudo de Jiang et al. ${ }^{14}$, seis pacientes com mucormicose foram tratados com anfotericina $B$ e cirurgia endoscópica e somente um deles foi a óbito por obstrução da artéria carótida interna.

A administração de anfotericina B aumenta a sobrevida de 37 para $79 \%$ em pacientes diabéticos e de zero para $47 \%$ nos não-diabéticos ${ }^{15}$. Para controle efetivo da doença, deve ser dada em doses elevadas, que variam de 0,8 a $1,5 \mathrm{mg} / \mathrm{kg} /$ $\mathrm{d}^{16}$, sendo que em altas doses é consideravelmente nefrotóxica. A associação de anfotericina B com sulfato de colesterol, 
também chamado "anfotericina B com dispersão coloidal" ou "lipossomal" é uma formulação capaz de liberar doses elevadas da droga com reduzida nefrotoxicidade devido à alteração na distribuição farmacológica, sendo assim uma opção no tratamento da mucormicose em pacientes refratário ao tratamento convencional. Tkatch et al. ${ }^{15}$ relataram um caso de mucormicose em paciente diabético em que foi utilizada a anfotericina B lipossomal com boa evolução. Alguns estudos mostram que a eficácia é a mesma entre o uso da anfotericina $B$ lipossomal e a tradicional anfotericina $B^{17,18}$. Rios-Gonçalves et al. ${ }^{19}$ relatam sobrevida de dois casos com tratamento com anfotericina $B$ seguido de tratamento cirúrgico. Outros autores recomendam anfotericina B lipossomal pois causa menos toxicidade permitindo uso de maiores doses, com melhores respostas ao tratamento ${ }^{20}$.

Uma outra alternativa é o uso de nistatina lipossomal, como relatado por Mileshkin et al. ${ }^{21} \mathrm{em}$ uma paciente com mucormicose refratária a tratamento com anfotericina $B$ lipossomal, na qual apresentou boa tolerância, sem evidência de nefrotoxicidade, com melhora clínica evidente.

0 uso adjuvante da câmera hiperbárica tem sido empregado em número pequeno de casos, com bons resultados. 0 oxigênio hiperbárico aumenta a tensão de oxigênio nos tecidos doentes que, em estudos in vitro, sugerem ter uma ação fungoestática22.

\section{RELATO DE CASO}

A.M.B., 51 anos, sexo feminino, natural de Piratininga SP, com antecedente de diabetes mellitus há 10 anos, em uso de insulina NPH $40 \mathrm{U}$ pela manhã e $30 \mathrm{U}$ à tarde, apresentou descompensação diabética - coma hiperosmolar - tendo sido internada na unidade de terapia intensiva com quadro de intensa dor abdominal. No seu segundo dia de internação, iniciou queixa de dor ocular esquerda importante, edema periorbital, proptose, febre $\left(39{ }^{\circ} \mathrm{C}\right)$ e saída de secreção fétida por trajeto fistuloso em região do canto interno ocular esquerdo (Figura 1), e evoluiu para amaurose do mesmo. Realizada tomografia de seios da face em cortes axiais e coronais, os quais identificaram importante aumento de partes moles junto à órbita esquerda entremeado por al guns pontos gasosos, associado ao encontro de área de realce capsular em lobo frontal, podendo corresponder a cerebrite (Figura 2 e 3 ). Frente ao quadro de descompensação da doença de base e abscesso orbital de evolução rápida foi realizada drenagem orbitária pela equipe da O ftalmologia. Pela persistência do quadro, foi abordada cirurgicamente dois meses depois em conjunto com a equipe da O torrinolaringologia, efetuando sinusectomia transmaxilar esquerda durante a qual foi visualizada secreção espessa branca nacarada em região etmoidal. No exame anatomo-patológico foi encontrado mucosa revestida por epitélio respiratório com processo inflamatório tendo em meio hifas septadas com diagnóstico final de zigomicose. Realizou tomografia de seios da face após o procedimento cirúrgico que revelou a manuten- ção da fístula naso-cutânea e material de atenuação de partes moles junto ao ápice da órbita e no interior do seio maxilar esquerdo (Figuras 4 e 5). Apesar de persistir com a fístula nasocutânea, apresentou progressiva melhora com uso de Anfotericina B (dose total $1.015 \mathrm{mg}$ ) e, após um mês de tratamento, a paciente não mais apresentava drenagem de secreção pela fístula. Encaminhada ao Serviço de Cirurgia Plástica e, após um ano sem qualquer sintoma, foi submetida à cirurgia estética na região naso-orbitária esquerda, estando a paciente atualmente satisfeita com aspecto facial (Figura 6).

\section{DISCUSSÃO}

A mucormicose geralmente afeta pacientes imunodeprimidos, como diabéticos em cetoacidose. 0 fungo em geral inicia sua invasão pela mucosa nasal, com acometimento dos seios da face, seguido de erosão do palato duro, órbita e cérebro ${ }^{17}$. Está no espectro oposto da sinusite fúngica alérgica, que é a forma mais comum de infecção fúngica sinusal, a qual acomete indivíduos "hipercompetentes" - atópicos ${ }^{6}$.

A progressão da doença leva a uma seqüência de sintomas que se inicia com dor facial súbita ou orbital unilateral, muitas vezes com obstrução nasal e secreção nasal necrótica. Pode ocorrer lesão lítica escura na mucosa nasal ou dorso do nariz, celulite orbitária e facial, febre, ptose palpebral, amaurose, oftalmoplegia, anestesia de córnea, terminando em coma e morte ${ }^{10}$.

A seqüência dos sintomas pode variar dependendo de onde iniciou a invasão do fungo e sua progressão está na dependência do estado imunológico do paciente. Em função das estruturas comprometidas, esta doença é divulgada com várias designações. Por exemplo: mucormicose cerebral, mucormicose craniofacial, mucormicose rino-órbito-cerebral, zigomicose rinocerebral, etc.

A nossa paciente apresentava comprometimento da fossa nasal, seios paranasais, órbita, periórbita, e pequena invasão cerebral, junto à fossa anterior. Quando há comprometimento da artéria carótida, o prognóstico é geralmente mais so mbrio ${ }^{11}$. Galetta et al. ${ }^{11}$ descreveram dois casos de mucormicose rinocerebral com trombose de artéria carótida interna com sobrevida. A definição de envolvimento orbital inclui alterações visuais, déficits da função dos músculos extraorbitários, oftalmoplegia, proptose ou celulite periorbitária ${ }^{9}$. A to mografia computado rizada é o exame de imagem mais apropriado para diagnóstico e acompanhamento da evolução de pacientes com suspeita de sinusite fúngica ${ }^{4}$, sendo que nesta paciente, este exame foi fundamental para se definir a extensão da lesão.

A primeira etapa no tratamento da sinusite fúngica invasiva é a compensação da doença de base ${ }^{3}$ que, no caso desta paciente do relato, foi a normalização de seus níveis glicêmicos.

Diagnóstico precoce e abordagem cirúrgica são os pontos mais importantes associados à boa evolução no tratamento de mucormicose ${ }^{15}$. Submetida a sinusectomia transmaxilar, foi 


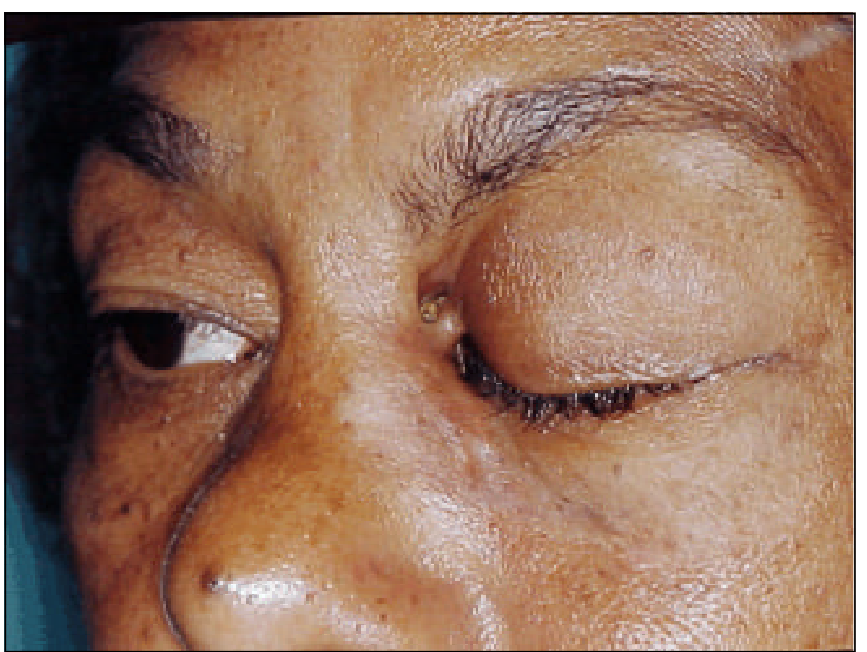

Figura 1. Retração de pele com fístula nasocutânea junto ao canto interno de órbita esquerda.

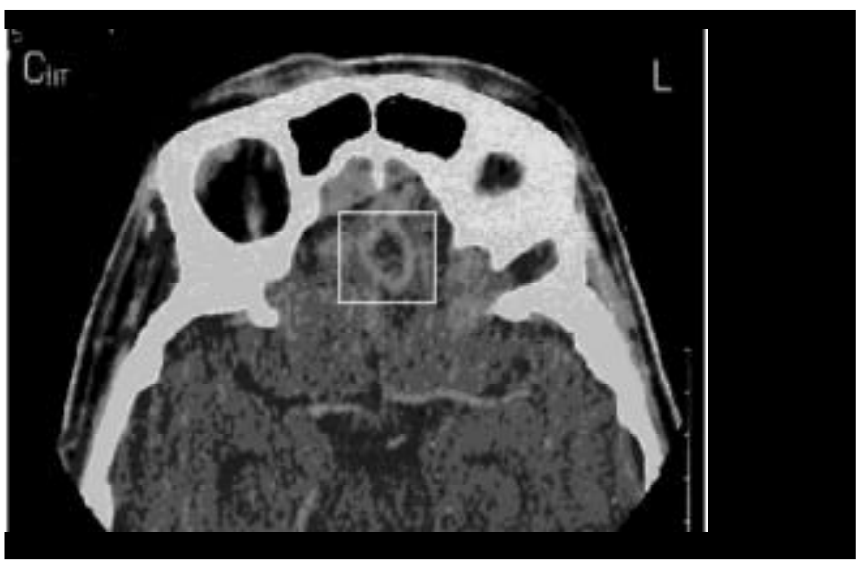

Figura 3. Corte axial evidenciando área de realce capsular no lobo frontal (cerebrite).

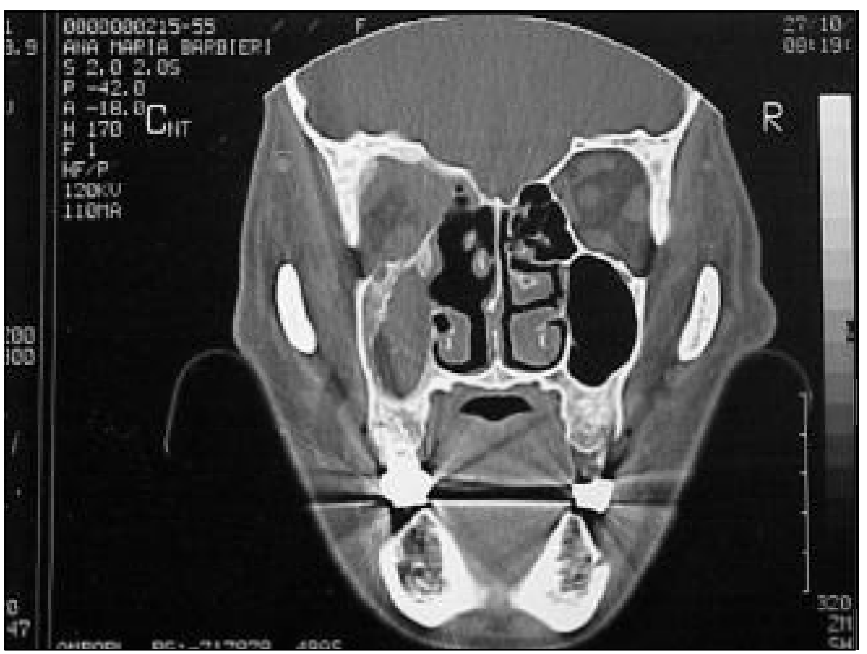

Figura 5. Tomografia computadorizada de seios da face - corte coronal. Pós-operatório:presença de material de partes moles no interior do seio maxilar esquerdo.

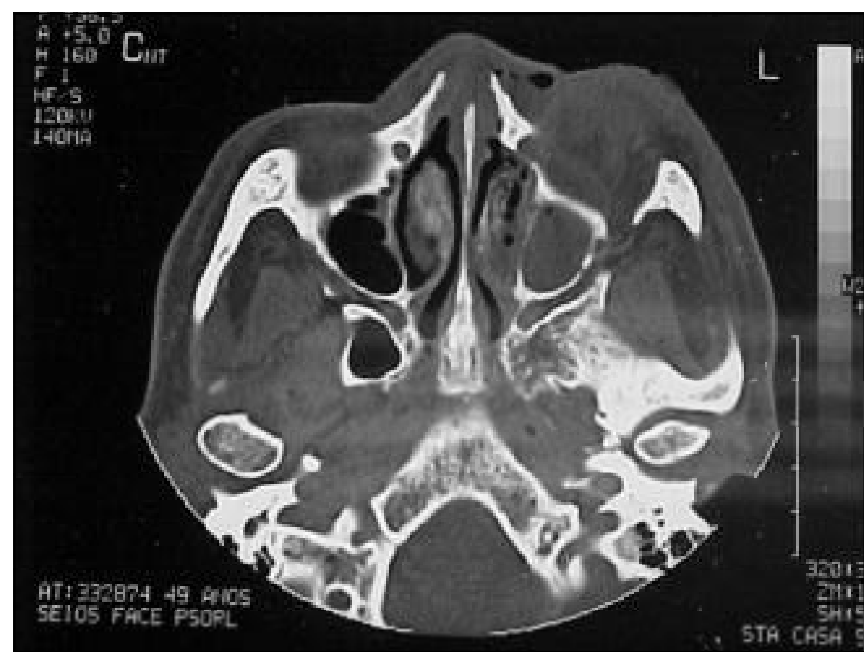

Figura 2. Corte axial mostrando importante assimetria facial com aumento de partes moles junto à órbita esquerda com alguns pontos gasosos em permeio.

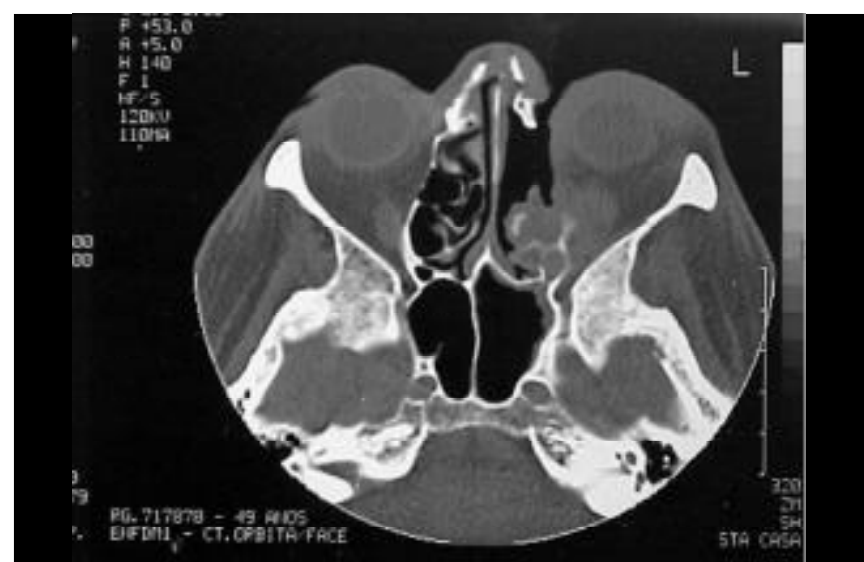

Figura 4. Tomografia computadorizada de seios da face - corte axial. Pós operatório mostrando fístula naso-cutânea e presença de material de partes moles junto ao ápice da órbita.

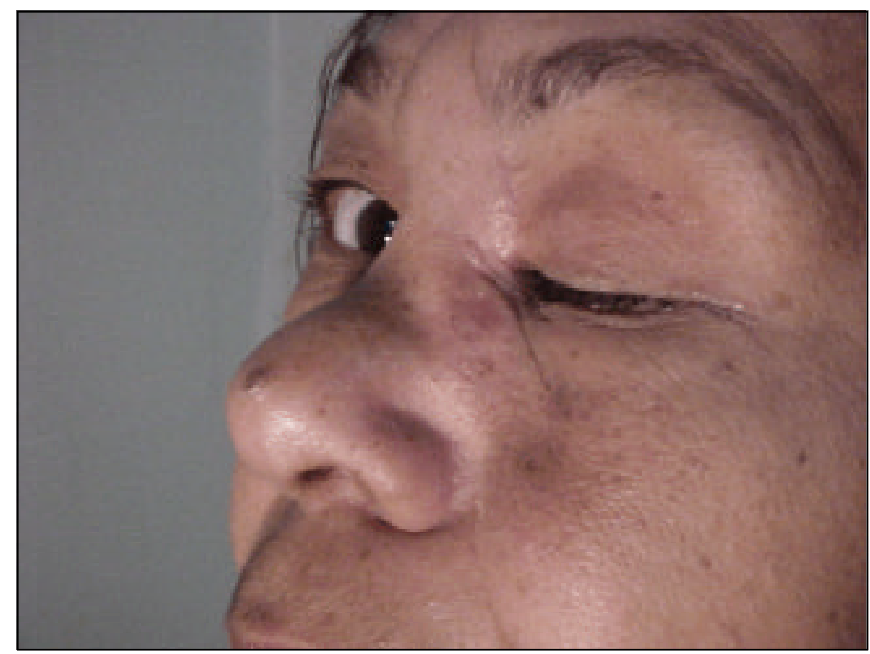

Figura 6. Paciente após fechamento da fístula naso-cutânea. 
observado muito material necrótico, e o anátomo-patológico confirmou a impressão clínica de zigomicose. Não se conseguiu identificar o agente no material da invasão tissular. Na avaliação pós-operatória havia dúvida quanto à exanteração da órbita esquerda, pois havia sinais de infecção resistente, porém como a literatura tem mostrado não haver diferença na evolução dos pacientes com conservação ou não da órbita, ela foi preservada ${ }^{1}$. A introdução de anfotericina $B$, aliada à antibioticoterapia que já vinha sendo utilizada, trouxe uma melhora do quadro clínico do paciente, permanecendo apenas a fístula nasocutânea, porém sem outros sintomas.

A introdução de anfotericina, segundo outros autores, tem favorecido a sobrevida dos pacientes portadores de mucormicose. Rios-Gonçalves et al. ${ }^{19}$, em 1991, acompanharam dois pacientes diabéticos com quadros clínicos semeIhantes ao da nossa paciente e que, com a mesma conduta, conseguiram êxito no tratamento. Também Santana et al. ${ }^{8}$, da mesma forma, divulgaram caso de mucormicose dos seios paranasais e orbitais com bom resultado. Segundo Mileshkin et al., uma alternativa é a anfotericina B lipossomal, com menos toxicidade permitindo atingir doses mais elevadas ${ }^{21}$. Leenders et al. ${ }^{20}$ realizaram um estudo multicêntrico randomizado comparando a eficácia da anfotericina B lipossomal e a anfotericina $B$ não lipossomal, e encontraram resultado superior naqueles pacientes tratados com a primeira delas.

A abordagem endoscópica pode também ser utilizada, mas é sempre necessário, no pré-operatório, tomografia computadorizada para identificar exatamente a extensão do processo infeccioso, pois, dependendo de sua extensão, se não é possível a remoção completa por via endoscópica ${ }^{14}$, é preferível a realização da cirurgia via aberta para o debridamento completo. Assim, em casos avançados em que há comprometimento da fissura pterigomaxilar ou envolvimento de órbita, fissura orbital inferior, fossa temporal e infratemporal, espaço bucal, o debridamento cirúrgico tradicional aberto deve ser realizado ${ }^{9}$. Como não há nenhum estudo comprovando que a exanteração orbital aumente a sobrevida, o olho somente deve ser removido quando há sinais de trombose de artéria retiniana, necrose de ápice orbital ou invasão orbitária ${ }^{1}$. Q ingli et al. ${ }^{12}$, no entanto, relataram um caso de mucormicose rino-cerebral com trombose de artéria retiniana e artéria ciliar nasal posterior no qual não realizaram exanteração da órbita, mas obtiveram bom resultado clínico final. Assim, na paciente deste relato, optamos por abordagem via aberta, mas sem exanteração de órbita.

\section{CONCLUSÃO}

A sobrevida da nossa paciente resultou de algumas ações combinadas iniciando-se com o imediato controle da descompensação da doença de base, e retirando-a do coma hiperosmolar. 0 debridamento cirúrgico abrangente e a utilização de anfotericina $B$, aliado ao pequeno envolvimento cerebral, foram fatores importantes no sucesso do tratamento.
A mucormicose deve ser considerada doença grave e potencialmente fatal e, por isso, é necessário que exista alto grau de suspeita frente sintomas sinusais agudos em imunodep rimidos, devendo estar a mucormicose entre os principais diagnósticos diferenciais.

\section{REFERÊNCIASBIBLIOGRÁFICAS}

1. Blitzer A, Lawson W. Fungal infections of the nose and paranasal sinuses. Otolaryngol Clin North Am 1993; 26 (6): 1007-35.

2. Ence BK, Gourley DS, Jorgensen NL. Allergic fungal sinusitis. Am J Rhinol 1990; 4: 169-78.

3. Ferguson BJ. Definitions of fungal rhinosinusitis. Otolaryngol Clin North Am 2000; 33(2): 227-35.

4. Stringer SP, Ryan MW. Chronic invasive fungal rhinosinusitis. Otolaryngol Clin North Am 2000; 33 (2): 375-87.

5 . Ferguson BJ. Fungus balls of the paranasal sinuses. Otolaryngol Clin North Am 2000; 33 (2): 389-98.

6. Houser SM, Corey JP. Allergic fungal rhinosinusitis pathophysiology epidemiology and diagnosis. Otolaryngol Clin North Am 2000; 33 (2): 399-409.

7. Bent JP, Kuhn FA. Antifungal activity against allergic fungal sinusitis organisms Laryngoscope 1996; 106: 1331-4.

8. Santana NOR, Pinheiro GB, Kehrle HM, Faria IP, Estrella CN Mucormicose de seios paranasais e órbitas em paciente imunocompetente: relato de caso e revisão da literatura. Rev Bra Otorrinolaringol 2001; 65(5) 727-30.

9. Alobid I, Bernal M, Calvo C, Vilaseca I, Berenguer J, Alós L. Treatment of rhinocerebral mucormycosis by combination of endoscopic sinus debridement and amphotericin B. Am J Rhinol 2001; 15: 327-31.

10. Yohia RA, Bullock JD, Aziz AA, Market RJ. Survival factors in rhinoorbital cerebral mucormycosis. Surv Ophthalmol 1994; 39: 3-22.

11. Galetta SL, Wulc AE, Goldberg HI, Nichols CW, Glaser JS. Rhinocerebral mucormycosis: mamagement and survival after carotid occlusion. Ann Neurol 1990; 28:103.

12. Qingli L, Orcutt JC, Seifter LS. Orbital mucormycosis with retinal and ciliary artery occlusions. $\mathrm{Br} J$ Ophthalmol 1989; 73:680-3.

13. Blokmanis A. Endoscopic diagnosis treatment and follow-up of tumors of the nose and sinuses. J Otolaryngol 1994; 23: 366-9.

14. Jiang RS, Hsu CY. Endoscopic sinus surgery for rhinocerebral mucormycosis. Am J Rhinol 1999; 13: 105-9.

15. Tkatch LS, Kusne S, Eibling D. Successful treatment of zygomycosis of the paranasal sinuses with surgical debridement and amphotericin B colloidal dispersion. Am J Otolaryngol1993; 14 (4): 249-53.

16. Sugar AM. Mucormycosis. Clin Infect Dis 1992; 14: S 126-9 (suppl 1).

17. Strasser MD, Kennedy RJ, Adam RD. Rhinocerebral mucormycosis therapy with amphotericin B lipid complex. Arch Intern Med 1961; 56: 337-9.

18. Fisher EW, Toma A, Fisher PH et al. Rhinocerebral mucormycosis: use of lipossomal anphotericin B. J Laryngol Otol 1991; 105: 575-7.

19. Rios-Gonçalves AJ, Rozenbaun R, Salles F, Laun IC, Mendes TP Medeiros P, Vieira ARM, Ramos DD, Chicralla C, Souza MOP, Londero AT. Mucormicose (Zigomicose) rinocerebral. Apresentação de dois casos com sobrevida. Arq Bras Med 1991; 65(3) 287-90.

20. Leenders $A C$, Daenen $S$, Jansen $R L$ et al. Liposomal amphotericin B compared with amphotericin B deoxycholate in the treatment of documented and suspected neutropenia- associated invasive fungal infections. $\mathrm{Br} J$ Haematol 1998; 103: 205-12.

21. Mileshkin L, Slavin M, Seymour JF, McKenzie A. Successful treatment of rhinocerebral zygomycosis using liposomal nystatin. Leukemia and Lynfoma 2001; 42(5): 1119-23.

22. Couch L, Theilen F, Mader JT. Rhinocerebral mucormycosis with cerebral extension successfully treated with adjuntive hyperbaric oxygen. Arch Otolaryngol 1988; 114: 791-4. 